\title{
Diffusive dark matter and dark energy scenario and $k$-essence in the context of Supernova Ia observations
}

\author{
Abhijit Bandyopadhyay 11 and Anirban Chatterję2 \\ Department of Physics \\ Ramakrishna Mission Vivekananda University \\ Belur Math, Howrah 711202, India
}

\begin{abstract}
We consider a unified model of interacting dark matter and dark energy to account for coincidence of present day dark energy and dark matter densities. We assume dark energy to be represented by a homogeneous scalar field $\phi$ whose dynamics is driven by a (noncanonical) $k$-essence Lagrangian with constant potential and the particles of dark matter fluid undergoing velocity diffusion in background medium of the $k$-essence scalar field $\phi$. This results in a transfer of energy from the fluid of dark matter to that of dark energy. This effect shows up as a source term in the continuity equation for dark matter and dark energy fluids. The source term involves a diffusion coefficient which is a measure of average energy transferred per unit time due to diffusion. We use time evolutions of the scale factor of background FRW spacetime, energy density and pressure of the dark fluid obtained from analysis of Supernova Ia data to obtain bounds on the diffusion parameter. For a constant potential in the $k$-essence Lagrangian, the temporal behaviour of a homogeneous $k$-essence field $\phi$ is obtained for different values of the diffusion parameter. The obtained temporal behaviour may be expressed as $\phi\left(t / t_{0}\right)=\phi_{0}+\varepsilon_{1}\left(t / t_{0}-1\right)+\varepsilon_{2}\left(t / t_{0}-1\right)^{2}$, where $t_{0}$ is the time corresponding to present epoch. The coefficients $\varepsilon_{1}$ and $\varepsilon_{2}$ have been found and obtained as linear functions of diffusion parameter.
\end{abstract}

\section{Introduction}

Measurement of luminosity distance of the type Ia Supernovae (SNe Ia) is the key observational ingredient in establishing the fact the universe has undergone a transition form decelerated to accelerated phase of expansion. This was first reported in 1998 independently by Riess et. al. [1] and Perlmutter et. al. [2]. Source of this late time cosmic acceleration is generally labelled as 'Dark Energy' (DE). Besides, observation of rotation curves of spiral galaxies [3], gravitational lensing [4], Bullet cluster [5] and other colliding clusters provide evidence for non-luminous matter in present universe manifesting its existence through gravitational interactions. Such 'matter', different from baryonic matter, is generally termed as 'Dark Matter' (DM). Measurements in satellite borne experiments - WMAP [6] and Planck [7] established that, dark energy and dark matter jointly contribute around $96 \%(\sim 69 \%$ dark energy and $\sim 27 \%$ dark matter) of total energy density of present universe. Rest $4 \%$ contribution comes from baryonic matter with negligible contribution from radiations. Despite countless searches for a physical theory of dark energy and dark matter, their origin and nature still remain a mystery. However, the $\Lambda-\mathrm{CDM}$ model [8] provides an excellent

\footnotetext{
${ }^{1}$ Email: abhijit@rkmvu.ac.in

${ }^{2}$ Email: anirban.chatterjee@rkmvu.ac.in
} 
agreement with a wide variety of cosmological data. Here 'CDM' denotes Cold Dark Matter content of the universe and $\Lambda$, the cosmological constant, represents dark energy of vacuum from viewpoint of particle physics. One of the major drawbacks of the model is the large disagreement between estimated energy density of vacuum and observed value of dark energy density. Also the same order of magnitude of observable values of dark matter and dark energy densities at present epoch seems accidental. Out of diverse theoretical attempts to resolve above mentioned problems, one interesting approach addresses the above two issues by considering a unified model of dark matter and dark energy [9, 10, 11, 12] where a dynamical relation between dark matter and dark energy was suggested to account for coincidence of present day dark energy and dark matter densities.

We assume dark energy to be represented by a homogeneous scalar field $\phi$ whose dynamics is driven by a $k$-essence Lagrangian with constant potential and the particles of dark matter fluid undergo velocity diffusion in background medium of the $k$-essence scalar field $\phi$. This results in a transfer of energy from the fluid of dark matter to that of dark energy. This effect shows up in the form of a non-zero source term in the continuity equation for the fluid of dark matter and dark energy as well where the source term involves a diffusion coefficient parameter which is a measure of average energy transferred per unit time due to diffusion. The continuity equation for dark matter fluid connects time evolution of dark matter energy density with the diffusion coefficient. Using luminosity distance and redshift measurements from observations of Supernova Ia (SNe Ia) we obtain temporal behaviour of certain cosmological parameters: scale factor $a(t)$ corresponding to Friedman-Robertson-Walker (FRW) spacetime background, total energy density and pressure of the dark fluid during late time phase of cosmic evolution. Using the results extracted from the SNe Ia data and the measured value of energy density of dark matter at present epoch from WMAP [6] and Planck [7] experiments, in the continuity equation for dark matter fluid we obtain the bounds on the diffusion coefficient parameter. We also obtain individual time dependences of energy densities of dark matter and dark energy components at different values of the diffusion parameter.

The dynamics $k$-essence scalar field $\phi$ playing the role of background medium in which diffusion takes place is governed by non-canonical Lagrangian of the form $L=V(\phi) F(X)$, where $X=(1 / 2) g^{\mu \nu} \nabla_{\mu} \phi \nabla_{\nu} \phi$ and the potential $V(\phi)$ is assumed constant. The constant potential in $k$-essence Lagrangian ensures a scaling relation $X(d F / d X)^{2}=C a^{-6}$ where $C$ is a constant. We identify the stress energy tensor corresponding to this Lagrangian with that of the dark energy fluid. Considering the scalar field $\phi$ to be homogeneous and exploiting the scaling relation we may directly relate the time derivative of scalar field with energy density and pressure of dark energy fluid. Using the temporal behaviour of dark energy density as obtained from our analysis we obtain temporal behaviour of the $k$-essence scalar field $\phi$ for different values of the diffusion coefficient. For any value of the diffusion coefficient within its allowed range, the obtained time $(t)$-dependence of the scalar field may be expressed as $\phi\left(t / t_{0}\right)=\phi_{0}+\varepsilon_{1}\left(t / t_{0}-1\right)+\varepsilon_{2}\left(t / t_{0}-1\right)^{2}$, where $t_{0}$ is the time corresponding to present epoch. The coefficients $\varepsilon_{1}$ and $\varepsilon_{2}$ are dependent on the value of diffusion parameter. Interestingly the observational data do not allow further higher order terms of $t$ in the obtained time dependence of field $\phi$ for any value of diffusion coefficient. Thus the $k$-essence scalar field $\phi$, in the context of this model, is found to have similar time evolution properties of quintessence scalar field that drives homogeneous inflation. Similar results in a different context were also obtained in [13]. 
In Sec. 2 we have discussed the basic frame work of the model of diffusive dark matter - dark energy interaction. In Sec. 3 we presented a brief description of the methodology of analysis of SNe Ia data and describe how time dependences of some relevant cosmological parameters extracted from the data has been exploited in the context of the model to obtain bounds on diffusion parameter. In Sec. 4 we have presented how we extracted the time evolution of the $k$-essence scalar field $\phi$ from the observational data, for a constant potential in $k$-essence Lagrangian. We summarised the results obtained in this work in Sec. 5 .

\section{Model of interacting diffusive dark energy and dark matter}

We indulge in a brief outline of the model of diffusive dark matter - dark energy interaction. The dynamics of cosmic evolution is governed by Einstein's equation

$$
R_{\mu \nu}-\frac{1}{2} g_{\mu \nu} R=8 \pi G T_{\mu \nu}
$$

where $g_{\mu \nu}$ is the spacetime metric, $R_{\mu \nu}$ is the Ricci tensor, $R=g^{\mu \nu} R_{\mu \nu}$ and $G$ is Newton's gravitation constant. The total energy-momentum tensor, $T_{\mu \nu}$, of the universe, is conserved: $\nabla^{\mu} T_{\mu \nu}=0$. We write $T_{\mu \nu}$ by decomposing it into contributions from the different constituent components (radiation $(R)$, baryonic matter $(b)$, dark matter $(\mathrm{dm})$ and dark energy $(\mathrm{de})$ ) of the universe as

$$
T_{\mu \nu}=T_{\mu \nu}^{R}+T_{\mu \nu}^{b}+T_{\mu \nu}^{\mathrm{dm}}+T_{\mu \nu}^{\mathrm{de}}
$$

When the dark sector (dark matter and dark energy) of the universe is not interacting with baryonic matter and radiation, the conservation of total energy momentum tensor implies $\nabla^{\mu}\left(T_{\mu \nu}^{R}+T_{\mu \nu}^{b}\right)=0$ and $\nabla^{\mu}\left(T_{\mu \nu}^{\mathrm{dm}}+T_{\mu \nu}^{\mathrm{de}}\right)=0$. Now for interacting dark matter and dark energy resulting in exchange of energy between then, we have

$$
\nabla_{\mu} T_{d e}^{\mu \nu}=-\nabla_{\mu} T_{d m}^{\mu \nu} \equiv-\sigma J^{\nu} .
$$

The transfer of energy between dark matter and dark energy is assumed to be caused by a diffusion in an ideal fluid environment. $\sigma(>0)$ denotes the diffusion coefficient which is a measure of average energy transferred to the particles of the dark matter fluid per unit time. $J^{\mu}$ denotes the current density of matter satisfying the conservation law $\nabla_{\mu} J^{\mu}=0$. We write the current density as $J^{\mu}=n u^{\mu}$, where $n$ is the number density of the particles of the dark matter fluid and $u^{\mu}$ represents the four-velocity of the fluid. In a homogeneous and isotropic spacetime background described by (FRW) metric with scale factor $a(t)$, taking $u^{\mu}=(1,0,0,0)$ for the comoving fluid, the conservation law $\nabla_{\mu} J^{\mu}=\nabla_{\mu}\left(n u^{\mu}\right)=0$ implies $n(t) a^{3}(t)=$ constant $=n_{0}$. Here $n_{0}$ denotes number density at present epoch and value of the scale factor at present epoch normalised to unity. Considering dark matter fluid to be an ideal fluid characterised by its energy density $\rho_{\mathrm{dm}}$ and pressure $p_{\mathrm{dm}}$, the energy momentum tensor $T_{d m}^{\mu \nu}$ is given by

$$
T_{d m}^{\mu \nu}=\rho_{\mathrm{dm}} u^{\mu} u^{\nu}+p_{\mathrm{dm}}\left(g^{\mu \nu}+u^{\mu} u^{\nu}\right)
$$

Using Eq. (41) and $J^{\nu}=n u^{\nu}$ in Eq. (3) and projecting it along the direction of $u^{\nu}$ we obtain

$$
\dot{\rho}_{\mathrm{dm}}+3 H \rho_{\mathrm{dm}}=\sigma \frac{n_{0}}{a(t)^{3}}
$$


where $H=\dot{a} / a$ is the Hubble parameter and we take dark matter as pressureless dust $\left(p_{\mathrm{dm}}=0\right)$. We also model the dark energy as an ideal fluid characterised by its energy density $\left(\rho_{\text {de }}\right)$ and pressure $\left(p_{\text {de }}\right)$. In FRW background spacetime, conservation of total energy momentum tensor for the dark fluid, $\nabla^{\mu}\left(T_{\mu \nu}^{\mathrm{dm}}+T_{\mu \nu}^{\mathrm{de}}\right)=0$, implies

$$
\left(\dot{\rho}_{\mathrm{dm}}+\dot{\rho}_{\mathrm{de}}\right)+3 H\left[\left(\rho_{\mathrm{dm}}+\rho_{\mathrm{de}}\right)+p_{\mathrm{de}}\right]=0 .
$$

From Eq. (5) and (6) we have

$$
\dot{\rho}_{\mathrm{de}}+3 H\left(\rho_{\mathrm{de}}+p_{\mathrm{de}}\right)=-\sigma \frac{n_{0}}{a(t)^{3}}
$$

\section{Bounds on the diffusion coefficient from SNe Ia data}

In this section we first discuss methodology to extract the temporal behaviour of the FRW scale factor $a(t)$, energy density and pressure of the dark fluid during the late time phase of cosmic evolution from the analysis of SNe Ia data. Then we discuss how we exploit the obtained time dependence of scale factor to find bounds on the diffusion coefficient term $\sigma n_{0}$ appearing in RHS of Eqs. (5) and (17).

The luminosity distance and redshift relationship for redshift values up to $z \sim 1$ obtained from SNe Ia observations is instrumental in revealing features of late time phase of cosmic evolution. At present there exists several dedicated and systematic searches and measurements of $\mathrm{SNe}$ Ia. These include supernova surveys in different redshift domains. The high redshift $(z \sim 1)$ projects are Supernova Legacy Survey (SNLS) (14, 15]) the ESSENCE project [16, the PanSTARRS survey ([17,,[18, [19]) Searches in redshift regime $0.05<z<0.4$ are performed in The SDSS-II supernova surveys $([20,[21],[22],[23],[24])$. Small redshift programmes $(z>0.1)$ include the Harvard-Smithsonian Center for Astrophysics survey (cFa) [25], the Carnegie Supernova Project $(\mathrm{CSP})([26,, 27,[28])$ the Lick Observatory Supernova Search (LOSS) [29] and the Nearby Supernova Factory (SNF) [30]. In all these surveys around one thousand SNe IA events were discovered. The measured luminosity distance has a very high statistical precision in the range between $z \sim 0.01$ and $z \sim 0.7$. Recently, "Joint Light-curve Analysis" (JLA) data ([18, [31, [32]) has been released. The data consists of $740 \mathrm{SNe}$ Ia events. This includes a new compilation of SNe Ia light curves including data from the full three years of the SDSS survey, first three seasons of the five-year SNLS survey and 14 very high redshift $0.7<z<1.4$ SNe Ia from space-based observations with the HST [33. This data sample has been extensively studied analysed in recent years. The data sets provide observed values of distance modulus at different measured values of red-shift $(z)$ We use the results of analysis of JLA data following flux-averaging technique described in [34, 35, 36] which takes care of different systematic uncertainties of SNe IA data in a elegant way. The $\chi^{2}$ function of JLA data is given by

$$
\chi^{2}=\sum_{i, j}\left(\mu_{\mathrm{obs}}-\mu_{\mathrm{th}}\right)_{i}\left(\sigma^{-1}\right)_{i j}\left(\mu_{\mathrm{obs}}-\mu_{\mathrm{th}}\right)_{j}
$$

where $\mu_{\mathrm{obs}}$ is the observed value of distance modulus at red-shift $z_{i}$. Theoretically, the distance modulus is related to the luminosity distance $d_{L}$ by $\mu_{\mathrm{th}}=5 \log _{10}\left[d_{L} / \mathrm{Mpc}\right]+25$ and is expressed as $\mu=m_{B}^{\star}-\left(M_{B}-\alpha \times X_{1}+\beta \times C\right)$ where $m_{B}^{\star}$ is the observed peak magnitude, 
$\alpha, \beta$ and $M_{B}$ are nuisance parameters. $X_{1}$ is the time stretching of the light-curve and $C$ describes the supernova color at maximum brightness as defined in detail in [37]. $\sigma$ is the covariant matrix involving statistical and systematic uncertainties as defined by Eq. (2.16) of [35]. For the details of calculation of $\chi^{2}$ we refer the reader to Refs. [35] and [36] where a comprehensive analysis of JLA data has been performed. A red-shift cut-off $\left(z_{\text {cut }}\right)$ is used there to separate out SN samples with $z<z_{\text {cut }}$ and $z \geq z_{\text {cut }}$. For samples with $z<z_{\text {cut }}$ the $\chi^{2}$ has been computed using Eq. (8) and for samples with $z \geq z_{\text {cut }}$ flux averaged values of $\mu$ and covariant matrix are used to compute $\chi^{2}$ in a way described in detail in [35]. For our work we take as input, the $z$-dependence of the function $E(z)=H(z) / H_{0}$, obtained from the marginalisation of $\chi^{2}$ over parameters $\alpha, \beta, M_{B}$ etc. as shown in left panel of Fig. 5 of Ref. [35]. We take $E(z)$ vs $z$ curve obtained in [35] for two benchmark cases: $z_{\text {cut }}=0$ and $z_{\text {cut }}=0.6$.

We may use the relations $H=\dot{a} / a$ and $a_{0} / a=1+z$ to write

$$
d t=-\frac{d z}{(1+z) H(z)}=-\frac{d z}{(1+z) H_{0} E(z)}
$$

The above equation on integration gives

$$
\frac{t(z)}{t_{0}}=1-\frac{1}{H_{0} t_{0}} \int_{z}^{0} \frac{d z^{\prime}}{\left(1+z^{\prime}\right) E\left(z^{\prime}\right)}
$$

where $t_{0}$ is the time denoting the present epoch. The function $E(z)$ as obtained from analysis of JLA data in [35] is used in Eq. (9), to obtain $t$ as a function of $z$ by performing the integration numerically. We then eliminate $z$ from the obtained $z-t(z)$ dependence and the the equation $a_{0} / a=1+z$ to obtain scale factor $a$ as a function of $t$.

In FRW spacetime background, the equations governing dynamics of late time cosmic evolution are the following two independent Friedmann equations

$$
\begin{aligned}
& H^{2}=\frac{8 \pi G}{3}\left(\rho_{\mathrm{de}}+\rho_{d m}\right) \\
& \frac{\ddot{a}}{a}=-\frac{4 \pi G}{3}\left[\left(\rho_{\mathrm{dm}}+\rho_{\mathrm{de}}\right)+3 p_{\mathrm{de}}\right]
\end{aligned}
$$

Equation of state of total dark fluid may then be expressed in terms of scale factor and its derivatives from above two equations as

$$
\omega=\frac{p_{\mathrm{de}}}{\rho_{\mathrm{de}}+\rho_{\mathrm{dm}}}=-\frac{2}{3} \frac{\ddot{a} a}{\dot{a}^{2}}-\frac{1}{3}
$$

We have considered a flat spacetime (zero curvature constant) and neglect contributions from radiation and baryonic matter during late time phase of cosmic evolution.

The time dependence of equation of state $\omega$ of the dark fluid may be obtained by using time dependence of scale factor in Eq. (12). We express the temporal behaviour in terms of a dimensionless time parameter $\tau$ defined as

$$
\tau=\ln a(t)
$$



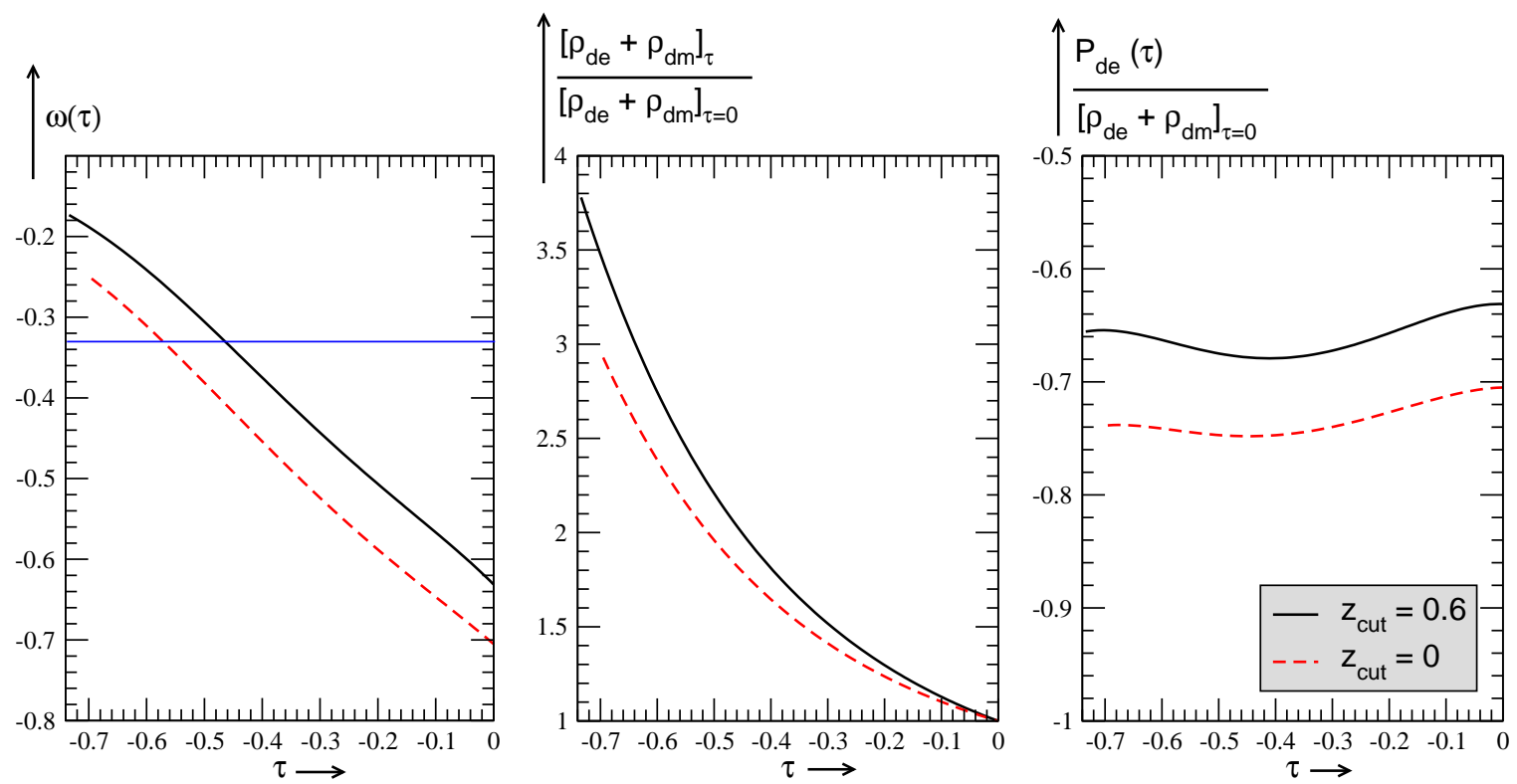

Figure 1: Left panel: Plot of $\omega(\tau)$ vs $\tau$ as obtained from analysis of observational data for $z_{\text {cut }}=0$ and $z_{\text {cut }}=0.6$ The horizontal line represent the value $\omega=-1 / 3(\ddot{a}=0)$, Middle panel: Plot of $\frac{\left[\rho_{\mathrm{de}}+\rho_{\mathrm{dm}}\right]_{\tau}}{\left[\rho_{\mathrm{de}}+\rho_{\mathrm{dm}}\right]_{0}}$ vs $\tau$ as obtained from analysis of observational data for $z_{\text {cut }}=0$ and $z_{\text {cut }}=0.6$, Right panel: Behaviour of $p_{\text {de }}$ vs $\tau$ for $Z_{\text {cut }}=0$ and $Z_{\text {cut }}=0.6$

The time domain accessible in Supernova Ia observations is $-0.7<\tau<0 . \tau=0$ corresponds to present epoch as the value of scale factor at present epoch is normalised to unity. In Fig. 1 we have shown time $(\tau)$-dependence of equation of state $\omega$. We find that the obtained dependence as shown in Fig. 1 may be fitted with a polynomial of the form

$$
\omega(\tau)=-1+\sum_{i=0} B_{i} \tau^{i}
$$

with coefficients $B_{i}$ 's given in Tab. 1.

\begin{tabular}{|lc|ll|c|}
\hline$B_{0}=$ & $-0.704(-0.631)$ & $B_{3}=$ & $-2.29(-3.76)$ & \\
\cline { 1 - 3 }$B_{1}=$ & $-0.61(-0.715)$ & $B_{4}=$ & $-2.81(-4.84)$ & \multirow{2}{*}{$\begin{array}{c}B_{i}=0 \\
\text { for } i>5\end{array}$} \\
\hline$B_{2}=$ & $-0.49(-1.04)$ & $B_{5}=$ & $-0.92(-1.93)$ & \\
\hline
\end{tabular}

Table 1: Values of $B_{i}$ 's in Eq. (14) providing best fit to the values of $\omega(\tau)$ extracted from SNe Ia data samples corresponding to $z_{\text {cut }}=0\left(z_{\text {cut }}=0.6\right)$.

In terms of the newly defined time parameter $\tau$, the continuity equation (66) for the total dark fluid may be written as

$$
\frac{d}{d \tau} \ln \left(\rho_{\mathrm{de}}+\rho_{\mathrm{dm}}\right)=-3(1+\omega(\tau)),
$$

which on integration gives

$$
\left[\rho_{\mathrm{de}}+\rho_{\mathrm{dm}}\right]_{\tau}=\left[\rho_{\mathrm{de}}+\rho_{\mathrm{dm}}\right]_{0} \exp \left[-3 \int_{\tau^{\prime}=0}^{\tau}\left(1+\omega\left(\tau^{\prime}\right)\right) d \tau^{\prime}\right]
$$




\begin{tabular}{|c|c|c|c|}
\hline$C_{0}=$ & 1 & $C_{3}=-0.65(-0.62)$ & \multirow{3}{*}{$\begin{array}{c}C_{i}=0 \\
\text { for } i>5\end{array}$} \\
\hline$C_{1}=$ & $-0.89(-1.10)$ & $C_{4}=1.36(2.096)$ & \\
\hline$C_{2}=$ & $1.28(1.65)$ & $-0.97(-1.05)$ & \\
\hline
\end{tabular}

Table 2: Values of $C_{i}$ 's in Eq. (17) providing best fit to the values of $\left(\rho_{\mathrm{de}}(\tau)+\rho_{\mathrm{dm}}(\tau) /\left[\rho_{\mathrm{de}}+\right.\right.$ $\left.\left.\rho_{\mathrm{dm}}\right]_{0}\right)$ extracted from SNe Ia data samples corresponding to $z_{\text {cut }}=0\left(z_{\text {cut }}=0.6\right)$.

\begin{tabular}{|cc|cc|c|}
\hline$\gamma_{0}=$ & $-0.705(-0.631)$ & $\gamma_{3}=$ & $-2.27(-3.463)$ & $\begin{array}{c}\gamma_{i}=0 \\
\text { for } i>4\end{array}$ \\
\cline { 1 - 2 }$\gamma_{1}=$ & $0.0016(-0.0319)$ & $\gamma_{4}=$ & $-1.48(-2.288)$ & \\
\cline { 1 - 1 }$\gamma_{2}=$ & $-0.93(-1.395)$ & & & \\
\hline
\end{tabular}

Table 3: Values of $\gamma_{i}$ 's in Eq. (19) providing best fit to the values of $\left(p_{\mathrm{de}}(\tau) /\left[\rho_{\mathrm{de}}+\rho_{\mathrm{dm}}\right]_{0}\right)$ extracted from SNe Ia data samples corresponding to $z_{\text {cut }}=0\left(z_{\text {cut }}=0.6\right)$.

Using the temporal dependence of the function $\omega(\tau)$ as obtained and depicted in Fig. 1. we perform the integration appearing on the right hand side of Eq. (16) numerically to obtain $\tau$-dependence of the total energy density of the dark fluid. The obtained temporal behaviour is shown in middle panel of Fig. 1. We find that this time dependence may be expressed in terms of a fitted polynomial of the form

$$
\left[\rho_{\mathrm{de}}+\rho_{\mathrm{dm}}\right]_{\tau}=\left[\rho_{\mathrm{de}}+\rho_{\mathrm{dm}}\right]_{0} \sum_{i=0} C_{i} \tau^{i}
$$

with coefficients $\left(C_{i}{ }^{\prime}\right.$ 's) given in Tab. 2 .

Using the obtained time dependences of $\omega$ and $\rho_{\mathrm{de}}+\rho_{\mathrm{de}}$ in Eq. (12),

$$
p_{\mathrm{de}}(\tau)=\omega(\tau)\left[\rho_{\mathrm{de}}+\rho_{\mathrm{de}}\right]_{\tau}
$$

we may also obtain the temporal behaviour of pressure of the dark energy fluid. The obtained dependence is shown in right panel of Fig. 1 and may be expressed in terms of a fitted polynomial

$$
p_{\mathrm{de}}(\tau)=\left[\rho_{\mathrm{de}}+\rho_{\mathrm{dm}}\right]_{0} \sum_{i=0} \gamma_{i} \tau^{i}
$$

with coefficients $\left(\gamma_{i}^{\prime}\right.$ 's) given in Tab. 3 ,

Time evolution of the dark matter energy density $\rho_{\mathrm{dm}}$ in this model is given by Eq. (5). In terms of time parameter $\tau$ the equation may be rewritten as

$$
\frac{d \rho_{\mathrm{dm}}}{d \tau}+3 \rho_{\mathrm{dm}}=\frac{\sigma n_{0}}{a^{3}(\tau) H(\tau)}
$$

Using $\tau$-dependence of the quantity $\frac{1}{a^{3} H}$ appearing in right hand side of above equation may be known using obtained temporal behaviour of the scale factor. We find that this dependence may be expressed in terms of a fitted polynomial

$$
\frac{1}{a^{3}(\tau) H(\tau)}=\sum_{i=0}^{5} D_{i} \tau^{i}
$$




\begin{tabular}{|cc|cc|c|}
\hline$D_{0}=$ & 1 & $D_{3}=$ & -2.70 & $D_{i}=0$ \\
\cline { 1 - 2 }$D_{1}=$ & -2.56 & $D_{4}=$ & -1.83 & for $i>5$ \\
\cline { 1 - 2 }$D_{2}=$ & 2.65 & $D_{5}=$ & -0.936 & \\
\hline
\end{tabular}

Table 4: Values of $D_{i}$ 's in Eq. (21) providing best fit to the values of $\frac{1}{a^{3}(\tau) H(\tau)}$ extracted from SNe Ia data samples corresponding to $z_{\text {cut }}=0$

with coefficients $\left(D_{i}\right.$ 's) given in Tab. 4. We now assume a series solution of Eq. (20) for $\rho_{\mathrm{dm}}$ as

$$
\rho_{\mathrm{dm}}=\left[\rho_{\mathrm{de}}+\rho_{\mathrm{dm}}\right]_{0} \sum_{i=0}^{\infty} \alpha_{i} \tau^{i}
$$

Substituting Eqs. (21) and (22) in Eq. (20) we obtain

$$
\sum_{i=0}^{\infty} i \alpha_{i} \tau^{i-1}+3 \sum_{i=0}^{\infty} \alpha_{i} \tau^{i}=K \sum_{i=0}^{5} D_{i} \tau^{i}
$$

where

$$
K=\frac{\sigma n_{0}}{\left[\rho_{\mathrm{de}}+\rho_{\mathrm{dm}}\right]_{0}}
$$

Equating the coefficients of $\tau^{i}$ from both sides of Eq. (23) we obtain

$$
\alpha_{i+1}=\frac{K D_{i}-3 \alpha_{i}}{i+1}
$$

Note that $K(>0)$ represents a diffusion parameter as it is linearly related to diffusion coefficient $\sigma$. We also note from Eq. (22) that $\alpha_{0}$ corresponds to the value of the fraction $\frac{\rho_{\mathrm{dm}}}{\left[\rho_{\mathrm{de}}+\rho_{\mathrm{dm}}\right]_{0}}$ at $\tau=0$ (present epoch). Mathematically, $\alpha_{0}$ is thus defined in the domain $0<\alpha<1$. Now for a given set of values for $\alpha_{0}$ and $K$, one may find $\alpha_{i}$ 's $(i>0)$ using the recursion relation (25). Since $D_{i}$ 's are zero for $i>5$ (see Tab. (4) and the term $(i+1)$ appears in the denominator of the recursion relation, the evaluated series $\left\{\alpha_{i}\right\}$ will always be convergent. Using these values of $\alpha_{i}$ 's we may compute $\rho_{\mathrm{dm}}$ at all values of $\tau$ from Eq. (22). Since $|\tau|<1$, and the series $\left\{\alpha_{i}\right\}$ is convergent, evaluated value of $\rho_{\mathrm{dm}}$ gets negligible contribution from terms above certain order in the summation series in Eq. (22). 3

The values of energy density $\left[\rho_{\mathrm{dm}}+\rho_{\mathrm{de}}\right]_{\tau}$ of the total dark fluid at any instant of time $\tau$ has been obtained directly from the analysis of SNe Ia data and shown in Fig. 1 (middle panel). The value of dark matter density $\rho_{\mathrm{dm}}\left(\tau ; \alpha_{0}, K\right)$ computed from Eq. (22) at any $\tau$, for a given $\left(\alpha_{0}, K\right)$ is subject to the constraint

$$
0<\rho_{\mathrm{dm}}\left(\tau ; \alpha_{0}, K\right)<\left[\rho_{\mathrm{dm}}+\rho_{\mathrm{de}}\right]_{\tau}
$$

\footnotetext{
${ }^{3}$ For example we find that values of $\rho_{\mathrm{dm}}$ computed with first 6 terms of the series and with first 100 terms of the series differ by less than 1 percent.
} 
for all values of $\tau$ in the range $(-0.7<\tau<0)$ accessible in SNe Ia observations. Imposition of the constraint (Eq. (26)) limits the range of allowed values of $\alpha_{0}$ and $K$. The shaded region in Fig. 2 depicts the allowed domain in $\alpha_{0}-K$ parameter space for which the constraint in Eq. (26) is realised.

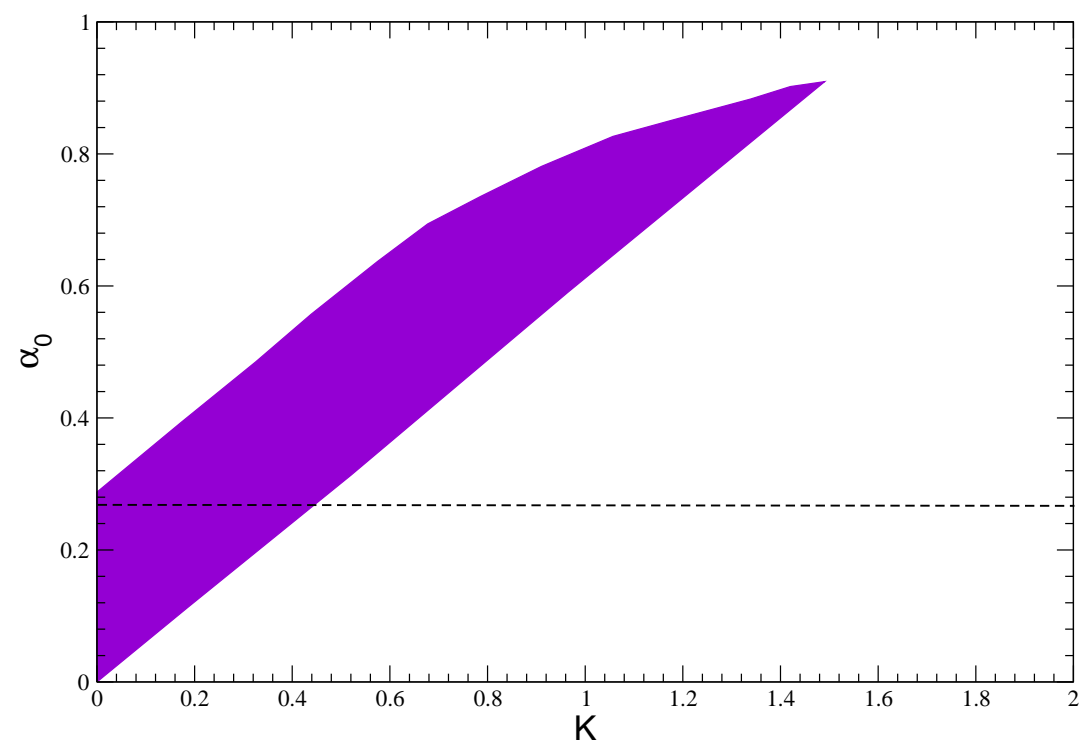

Figure 2: Region of $\alpha_{0}-K$ parameter space

However, measurements in satellite borne experiments - WMAP [6] and Planck [7] established that fractional contribution of dark matter to the total energy density of present universe is $\sim 0.27$. So neglecting contributions from radiation and baryonic matter to the total energy density of present universe, we may take measured value of $\alpha_{0}$ to be $\sim 0.27$. This has been depicted by a horizontal line in Fig. 2. This value of $\alpha_{0}$ corresponds to an allowed range of diffusion parameter $K$ as $0 \leqslant K<0.44$.

\section{Diffusive dark energy - dark matter scenario with dark en- ergy as a $k$-essence scalar field}

In this section we realise dark energy in terms of a $k$-essence scalar field and investigate its implications in the context of diffusive dark matter - dark energy model. We assume dark energy to be represented by a homogeneous scalar field $\phi$ whose dynamics is driven by a $k$-essence Lagrangian with constant potential. Using the temporal behaviour of scale factor, total energy density of dark fluid, pressure of the dark energy fluid, as extracted from SNe Ia data, we find nature of time dependence of the scalar field $\phi$. These has been obtained for different values of the diffusion coefficient, $K$ within it's range allowed from SNe Ia data as obtained in Sec. 3. The dependence of the field on diffusion coefficient has also been obtained.

The $k$-essence models involve non-canonical Lagrangian of the form $L=V(\phi) F(X)$ where $X=(1 / 2) g^{\mu \nu} \nabla_{\mu} \phi \nabla_{\nu} \phi, F$ and $V$ are functions of $X$ and $\phi$ respectively. The stress energy 
tensor corresponding to this Lagrangian is equivalent to that of an ideal fluid with energy density $V(\phi)\left(2 X F_{X}-F\right)$ and pressure $V(\phi) F(X)$ respectively, where $F_{X}=d F / d X$. Identifying this fluid as dark energy we write

$$
\begin{aligned}
& p_{\mathrm{de}}=V F(X), \\
& \rho_{\mathrm{de}}=V\left(2 X F_{X}-F\right) .
\end{aligned}
$$

Here we consider the $k$-essence model with constant potential, $V(\phi)=V$ and the scalar field $\phi$ in FRW spacetime background to be homogeneous: $\phi(x) \equiv \phi(t)$. We then have $X=(1 / 2) \dot{\phi}^{2}$ and constancy of the potential $V$ ensures existence of scaling relation [38, 39]

$$
X F_{X}^{2}=C a^{-6}, \quad C \text { is a constant }
$$

From Eqs. (27) and (28) we have

$$
\rho_{\mathrm{de}}+p_{\mathrm{de}}=2 V X F_{X}
$$

Eliminating $X$ from Eqs. (29) and (30) we obtain

$$
\frac{F_{X}}{2 C V}=\frac{a^{-6}}{\rho_{\mathrm{de}}+p_{\mathrm{de}}}
$$

Eliminating $F_{X}$ from Eq. (29) and Eq. (31) we obtain

$$
X=\frac{a^{6}\left(\rho_{\mathrm{de}}+p_{\mathrm{de}}\right)^{2}}{4 C V^{2}}
$$

For a homogeneous $k$-essence field $\phi$, we have $X=\frac{1}{2} \dot{\phi}^{2}$. Changing the time parameter from $t$ to $\tau$ we may write

$$
X=\frac{1}{2}\left[H\left(\frac{d \phi}{d \tau}\right)\right]^{2}
$$

From Eq. (32) and (33) we have

$$
\left[\frac{\sqrt{2 C} V}{\left(\rho_{\mathrm{dm}}^{0}+\rho_{\mathrm{de}}^{0}\right)}\right]\left(\frac{d \phi}{d \tau}\right)=\frac{a^{3}}{H}\left[\frac{\rho_{\mathrm{de}}}{\left(\rho_{\mathrm{dm}}^{0}+\rho_{\mathrm{de}}^{0}\right)}+\frac{p_{\mathrm{de}}}{\left(\rho_{\mathrm{dm}}^{0}+\rho_{\mathrm{de}}^{0}\right)}\right]
$$

Eq. (34) on Integration gives

$$
\left[\frac{\sqrt{2 C} V}{\left(\rho_{\mathrm{dm}}^{0}+\rho_{\mathrm{de}}^{0}\right)}\right]\left(\phi(\tau, K)-\phi_{0}(K)\right)=\int_{\tau^{\prime}=0}^{\tau} d \tau^{\prime}\left[\frac{a^{3}\left(\tau^{\prime}\right)}{H\left(\tau^{\prime}\right)}\left(\frac{\rho_{\mathrm{de}}\left(\tau^{\prime}\right)}{\rho_{\mathrm{dm}}^{0}+\rho_{\mathrm{de}}^{0}}+\frac{p_{\mathrm{de}}\left(\tau^{\prime}\right)}{\rho_{\mathrm{dm}}^{0}+\rho_{\mathrm{de}}^{0}}\right)\right]
$$

The temporal behaviour of the quantities $\left[\rho_{\mathrm{dm}}+\rho_{\mathrm{de}}\right]_{\tau} /\left(\rho_{\mathrm{dm}}^{0}+\rho_{\mathrm{de}}^{0}\right)$ and $p_{\mathrm{de}}(\tau) /\left(\rho_{\mathrm{dm}}^{0}+\rho_{\mathrm{de}}^{0}\right)$ extracted from SNe Ia observations have been shown in Fig. 1. As discussed in Sec. 3, using Eq. (22) we may compute dark matter density $\rho_{\mathrm{dm}}\left(\tau ; \alpha_{0}, K\right) /\left(\rho_{\mathrm{dm}}^{0}+\rho_{\mathrm{de}}^{0}\right)$ corresponding to a set of values of parameters $\left(\alpha_{0}, K\right)$ within their allowed domain depicted in Fig. 2, The dark energy density $\rho_{\text {de }}$ may also be evaluated at a given $\left(\alpha_{0}, K\right)$ value as

$$
\frac{\rho_{\mathrm{de}}\left(\tau ; \alpha_{0}, K\right)}{\left(\rho_{\mathrm{dm}}^{0}+\rho_{\mathrm{de}}^{0}\right)}=\frac{\left[\rho_{\mathrm{dm}}+\rho_{\mathrm{de}}\right]_{\tau}}{\left(\rho_{\mathrm{dm}}^{0}+\rho_{\mathrm{de}}^{0}\right)}-\frac{\rho_{\mathrm{dm}}\left(\tau ; \alpha_{0}, K\right)}{\left(\rho_{\mathrm{dm}}^{0}+\rho_{\mathrm{de}}^{0}\right)}
$$




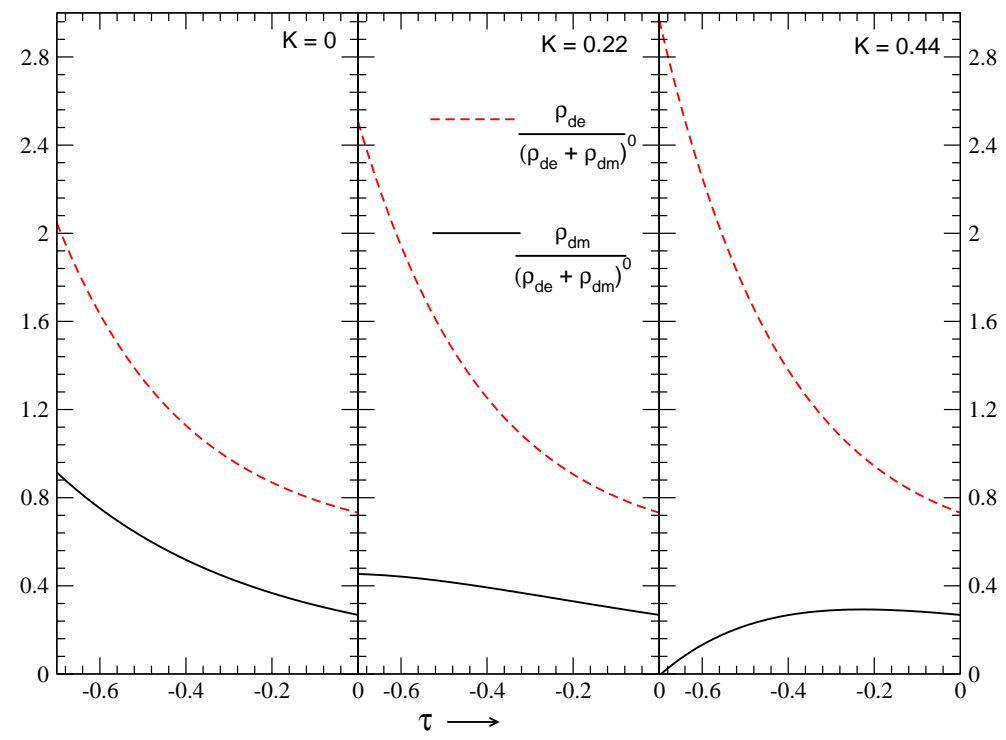

Figure 3: Temporal behaviour for dark matter density $\rho_{\mathrm{dm}}$ and dark energy density $\rho_{\mathrm{de}}$ for three benchmark values of diffusion parameter: $K=0,0.22,0.44$

We compute $\rho_{\mathrm{de}}$ from above equation for $\alpha_{0} \equiv \rho_{\mathrm{dm}}^{0} /\left(\rho_{\mathrm{dm}}^{0}+\rho_{\mathrm{de}}^{0}\right)=0.27$ (which is close to the experimentally observed value) and for different values of $K$ in the corresponding allowed range $0<K<0.44$. The obtained temporal behaviour for dark matter density $\rho_{\mathrm{dm}}$ and dark energy density $\rho_{\text {de }}$ for three benchmark values of diffusion parameter $K(0,0.22$ and 0.44$)$ are shown in Fig. 3. With the obtained $\tau$ dependences of scale factor $a$, Hubble parameter $H$, $p_{\text {de }}(\tau)$ and $\rho_{\text {de }}(\tau, K)$, we compute values of integrand appearing in the right hand side of Eq. (35) at different values of $\tau$ and $K$. We then perform the integration numerically to obtain temporal behaviour of the $k$-essence scalar field for different values of the diffusion parameter $K$. The results are shown in Fig. 4. We have shown the time dependence in terms of both the time parameters $\tau=\ln a(t)$ and $t$. We find that, for any value of the diffusion parameter $K$, the time dependence of the $k$-essence scalar field $\phi$ may be fitted in terms of polynomial of degree 2 as

$$
\phi\left(t / t_{0}\right)=\phi_{0}+\varepsilon_{1}\left(t / t_{0}-1\right)+\varepsilon_{2}\left(t / t_{0}-1\right)^{2}
$$

where $\phi_{0}$ is the value of the field at present epoch $\left(\tau=0\right.$ or $\left.t / t_{0}=1\right)$. The coefficients $\varepsilon_{1}$ and $\varepsilon_{2}$ depend on chosen value of $K$. From the analysis we find both these dependences to be linear in $K$ and are given by

$$
\begin{aligned}
& \varepsilon_{1}(K)=-0.64+0.67 K \\
& \varepsilon_{2}(K)=-0.80+0.45 K
\end{aligned}
$$

The field $\phi$ thus has a linear dependence of diffusion parameter $K$.

\section{Conclusion}

In this work we have considered a model of diffusive dark matter and dark energy where dark energy is represented by a homogeneous $k$-essence scalar field $\phi$ with a (non-canonical) 


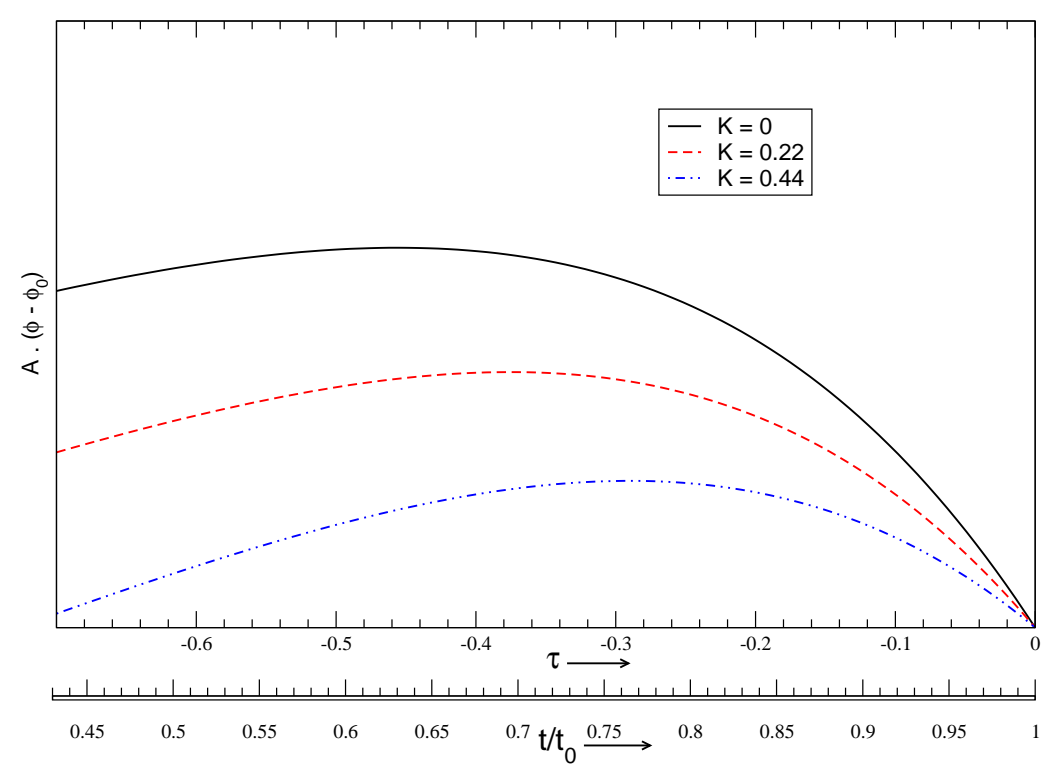

Figure 4: Temporal behaviour of the quantity $A\left(\phi-\phi_{0}\right)$, (where $A \equiv \sqrt{2 C} V /\left(\rho_{\mathrm{dm}}^{0}+\rho_{\mathrm{de}}^{0}\right)$ is a constant) for three benchmark values of diffusion parameter: $K=0,0.22,0.44$.

Lagrangian with constant potential. We assume particles of the dark matter fluid undergoing velocity diffusion in the background medium of the $k$-essence scalar field $\phi$. This diffusion establishes a dynamical relation between dark matter and dark energy causing energy transfer from dark matter to the background $k$-essence field. Motivations behind considering such models are to account for coincidence of measured values present day dark energy and dark matter densities. We have shown that this model is supported by luminosity distance and redshift data from SNe Ia observations. The energy transfer between dark matter and background $k$-essence field $\phi$ representing dark energy shows up as a source term in the continuity equations for the individual fluids of dark matter and dark energy. The source term is proportional to a diffusion coefficient which is a measure of average energy transfer per unit time from dark matter to dark energy. In this work we realise this quantity in terms of a dimension diffusion parameter $k$ introduced in Eq. (24). Using time dependences of energy density and pressure of the dark fluid as extracted from SNe Ia observations and the result that dark matter contributes $\sim 27 \%$ of the total energy density of present universe, we obtain a constrain on the value of the diffusion parameter $K$ as $0 \leqslant K<0.44$.

Temporal behaviour of dark matter and dark energy densities in this diffusive interaction scenario for different values of diffusion parameter $(K)$ are shown in Fig. 3. $K=0$ corresponds to non-interacting dark matter and dark energy. Then Energy density of dark matter that satisfies the continuity equation $\dot{\rho}_{\mathrm{dm}}+3 H \rho_{\mathrm{dm}}=0$ and $\rho_{\mathrm{dm}} \sim a^{-3}$. Non-zero (positive) values of $K$ corresponds to energy transfer from dark matter to the dark energy field $\phi$ and temporal behaviour of $\rho_{\mathrm{dm}}$ differs from that of $a^{-3}$. Higher values of $K$ corresponds to higher average value of transferred energy from dark matter fluid to dark energy fluid. At any given epoch, dark matter energy density is therefore always lower for higher values of $K$. Plots of Fig. 3 depict this feature. 
We assume the $k$-essence scalar field $\phi$ representing dark energy to be homogeneous and described by a non-canonical Lagrangian with constant potential which ensures existence of a scaling relation Eq. (29). Using the scaling relation we described a methodology to obtain the temporal behaviour of the field $\phi$ without prior knowledge of function $F(X)$ in $K$-essence Lagrangian. The dependence of the field $\phi$ on the diffusion parameter $K$ is also obtained. The obtained temporal behaviour of the field is expressed as $\phi\left(t / t_{0}\right)=\phi_{0}+\varepsilon_{1}\left(t / t_{0}-1\right)+$ $\varepsilon_{2}\left(t / t_{0}-1\right)^{2}$. where $t_{0}$ is the time corresponding to present epoch. The coefficients $\varepsilon_{1}$ and $\varepsilon_{2}$ are functions of diffusion parameter $K$ and the obtained dependence is expressed in Eq. (38). We observe that SNe Ia data do not allow any room for terms of $t$ having order more than 2 , independent of the values of diffusion parameter $K$. Thus the kessence field $\phi$ has similar temporal behaviour as that of quintessence scalar field which is responsible for homogeneous inflation.

\section{References}

[1] A. G. Riess et al., Astron. J. 116, 1009 (1998)

[2] S. Perlmutter et al., Astrophys. J. 517, 565 (1999)

[3] Y. Sofue and V. Rubin, Ann. Rev. Astron. Astrophys. 39, 137 (2001).

[4] M. Bartelmann and P. Schneider, Phys. Rept. 340, 291 (2001).

[5] D. Clowe, A. Gonzalez and M. Markevitch Astrophys. J. 604, 596 (2004).

[6] G. Hinshaw et al. [WMAP Collaboration], Astrophys. J. Suppl. 208, 19 (2013).

[7] P. A. R. Ade et al. [Planck Collaboration], Astron. Astrophys. 571, A16 (2014).

[8] S. Weinberg, Rev. Mod. Phys. 61, 1 (1989)

[9] M. Szydowski and A. Stachowski, Phys. Rev. D 94, no. 4, 043521 (2016)

[10] S. Calogero, JCAP 1111 (2011) 016

[11] S. Calogero, J. Geom. Phys. 62 (2012) 22082213

[12] Z. Haba, A. Stachowski and M. Szydowski, JCAP 1607, no. 07, 024 (2016)

[13] A. Bandyopadhyay, D. Gangopadhyay and A. Moulik, Eur. Phys. J. C 72, 1943 (2012)

[14] Astier et al., A \& A, 447, 31 (2006)

[15] Sullivan et al., ApJ, 737, 102 (2011)

[16] Wood-Vasey et al., ApJ , 666 , 694 (2007)

[17] Tonry et al., ApJ , 750 , 99 (2012)

[18] Scolnic et al., ApJ submitted arXiv:1310.3824

[19] Rest et al., ApJ, submitted, arXiv:1310.3824 
[20] Frieman et al., AJ, 135 ,338 (2008)

[21] Kessler et al., ApJS , 185 , 32 ( 2009a)

[22] Sollerman et al., ApJ , 703 ,1374 (2009)

[23] Lampeitl et al., MNRAS , 401 , 2331 (2010a)

[24] Campbell et al., ApJ , 763 ,88 (2013)

[25] Hicken et al., ApJ , 700 , 331 (2009)

[26] Contreras et al., AJ , 139 , 519 (2010)

[27] Folatelli et al., AJ , 139 , 120 (2010)

[28] Stritzinger et al., AJ , 142 , 156 (2011)

[29] Ganeshalingam et al., MNRAS , 433 , 2240 (2013)

[30] Aldering et al., SPIE Conf. Ser , 4836 , 61

[31] Conley et al., ApJS , 192 , 1 (2011)

[32] Suzuki et al., ApJ , $\mathbf{7 4 6 ~ , ~} 85$ (2012)

[33] Riess et al., ApJ , 659 , 98 (2007)

[34] Y. Wang, Astrophysical Journal 536 (2000) 531

[35] S. Wang, S. Wen and M. Li, JCAP 1703, no. 03, 037 (2017)

[36] S. Wang, Y. Wang, Phys. Rev. D 88 (2013a) 043511.

[37] M. Betoule, R. Kessler, J. Guy, et al., Astron. Astrophys 568 (2014) A22.

[38] R. J. Scherrer, Phys. Rev. Lett. 93011301 (2004)

[39] L. P. Chimento, Phys. Rev. D69 123517 (2004) 